\title{
The round table approach in infective endocarditis $\&$ cardiovascular implantable electronic devices infections: make your e-Team come true
}

\author{
Paola A. Erba ${ }^{1}$ - Gilbert Habib ${ }^{2,3}$ • Andor W. J. M. Glaudemans ${ }^{4}$ - Jose M. Miro ${ }^{5}$. \\ Riemer H. J. A. Slart ${ }^{4}$
}

Published online: 7 April 2017

(C) Springer-Verlag Berlin Heidelberg 2017

Over the past decade health care systems have faced a set of challenges leading to an increasing emphasis on interdisciplinary team work. Workforce re-structuring is the result of several factors including population changes and growth in medical knowledge with the consequent increasing rate of specialization. Aging of the population, the increasing numbers of patients with more complex needs, associated with chronic diseases, has increased tremendously. On the other side, increasing specialization within health professions have led to fragmentation of disciplinary knowledge resulting in no one health care professional being able to meet all the complex needs of their patients. Multidisciplinary team working has been proposed as the model for several years in oncology in many hospitals and medical centres. More recently, the multidisciplinary team approach has been extended in patient management as a response to manage complex disease states, beyond oncology. In the Cardiology field, the transcatheter aortic valve implantation (TAVI) team is a recent example [1]. In the field of infective endocarditis (IE), in some centers a multidisciplinary approach for evaluating patients with IE is

Paola A. Erba

paola.erba@unipi.it

1 Department of Nuclear Medicine, Azienda Ospedaliero-Universitaria Pisana, Pisa, Italy

2 Aix-Marseille Universite, URMITE, Marseille, France

3 Cardiology Department, APHM, La Timone Hospital, Marseille, France

4 Department of Nuclear Medicine and Molecular Imaging, Medical Imaging Center, University Medical Center Groningen, University of Groningen, Groningen, The Netherlands

5 Infectious Diseases Service, Hospital Clinic - IDIBAPS, University of Barcelona, Barcelona, Spain being used for many years in order to improve management and outcomes [2]. Recently, in the newest update of the European Society of Cardiology (ESC) Guideline for the management of IE this multidisciplinary "round table" approach for the diagnosis and treatment of this complex lifethreatening disease has been included [3]. The 2015 ESC Guidelines recommend the constitution of an Endocarditis Team (E-Team), a multidisciplinary "round table" system involving specialists in imaging, cardiologists, cardiac surgeons, infectious disease specialists, microbiologists and others (Fig. 1). This round table approach has been shown to significantly reduce the in-hospital and 1- and 3-year mortality in France, Italy and Spain [4-6]. To be effective, the structure of an E-Team has to be modelled, taking specifications of health systems, including cultural and socio-economics, in consideration. Its success is contingent upon "knowledge of one's own area as well as other team members' disciplines, flexibility of roles, and comfort and skill in supplying and receiving education between the disciplines" [7]. To promote effective collaboration, the team must address issues of group dynamics, including role clarification, team unity, communication, and patterns of decision-making and leadership, as underlined recently $[8,9]$. A communication framework, which supports and ensures interactive participation from all relevant team members at regular clinical meetings needs to be established to achieve integrated diagnostic-therapeutic strategies (using the current guidelines), (inter)national registries, organized follow-up of the patients, and control by training \& certification (Fig. 1). Finally, the E-team must define the quality control measures to audit their activities according to prespecified criteria [2].

By putting imaging in a central position in the diagnostic algorithm of patients with suspected IE, the 2015 ESC Guidelines recognise the importance within the E-Team of a new entity, the "Clinical Imaging Specialist": specialists in 
Fig. $1 I E$ infective endocarditis, CIED cardiovascular implantable electronic device

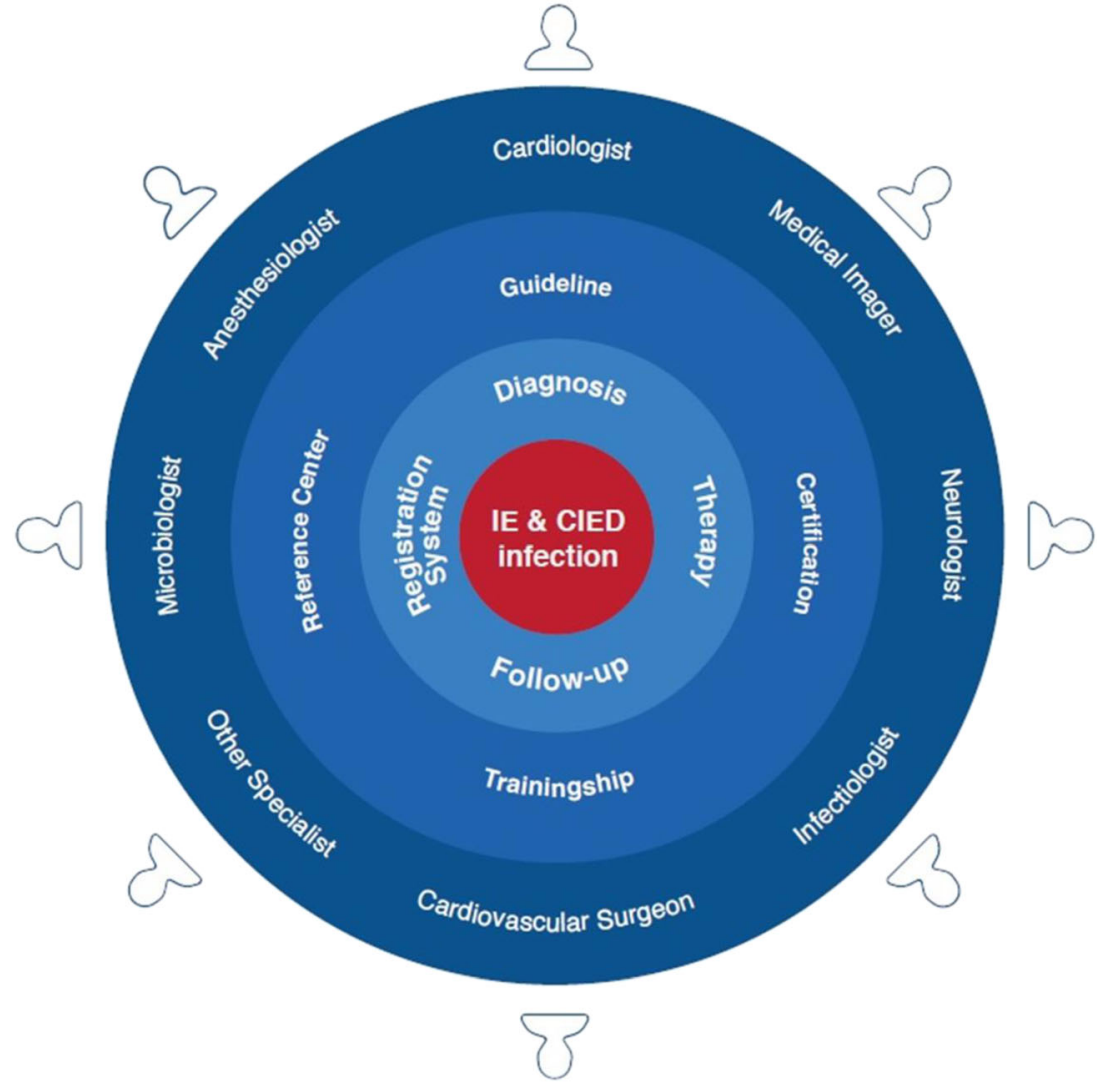

echocardiography, but also experts in multimodality medicine, including nuclear molecular imaging, cardiac CT, and MRI. The Clinical Imager plays an active role in the teamwork program and in the global educational planning, developing "capabilities" and "competencies", core skills, knowledge and attitudes to facilitate inter-specialist communications.

The round table approach for all its facets is indispensable for the integrated diagnostic-therapeutic strategy in IE and CIED infections, and the time has come to make the E-Team come true. The challenge of the "Clinical Imager" within the E-Team is to establish a new professional perspective: a new vision of the "Imager", no longer thinking as an individual, but as a part and contributor to the team, translating the image content into a clinical planning and decision-making process to deliver quality patient care within such complex contexts.

\section{References}

1. Agarwal S, Tuzcu EM, Krishnaswamy A, Schoenhagen P, Stewart WJ, Svensson LG, et al. Transcatheter aortic valve replacement: current perspectives and future implications. Heart. 2015;101(3): 169-77.

2. Mestres CA, Paré JC, Miró JM, Working Group on Infective Endocarditis of the Hospital Clínic de Barcelona. Organization and functioning of a multidisciplinary team for the diagnosis and treatment of infective endocarditis: a 30-year perspective (19852014). Rev Esp Cardiol. 2015;68(5):363-8.

3. Habib G, Lancellotti P, Antunes MJ, et al. 2015 ESC guidelines for the management of infective endocarditis: the task force for the management of infective endocarditis of the European Society of Cardiology (ESC) endorsed by: European Association for CardioThoracic Surgery (EACTS), the European Association of Nuclear Medicine (EANM). Eur Heart J. 2015;36:3075-128.

4. Botelho-Nevers E, Thuny F, Casalta JP, Richet H, Gouriet F, Collart $\mathrm{F}$, et al. Dramatic reduction in infective endocarditis-related mortality with a management-based approach. Arch Intern Med. 2009;169(14):1290-8.

5. Chirillo F, Scotton P, Rocco F, et al. Impact of a multidisciplinary management strategy on the outcome of patients with native valve infective endocarditis. Am J Cardiol. 2013;112(8):1171-6. doi:10. 1016/j.amjcard.2013.05.060.

6. Carrasco-Chinchilla F, Sánchez-Espín G, Ruiz-Morales J, Rodríguez-Bailón I, Melero-Tejedor JM, Ivanova-Georgieva R, et al. Influence of a multidisciplinary alert strategy on mortality due to left-sided infective endocarditis. Rev Esp Cardiol. 2014;67(5):380-6.

7. Ducanis AJ, Golin AK. The interdisciplinary health care team: a handbook. Aspen Publishers; 1979.

8. Gomes A, Glaudemans AW, Touw DJ, et al. Diagnostic value of imaging in infective endocarditis: a systematic review. Lancet Infect Dis. 2017;17(1):e1-e14. doi:10.1016/S1473-3099(16) 30141-4. Review.

9. Cahill TJ, Baddour LM, Habib G, Hoen B, Salaun E, Pettersson GB, et al. Challenges in infective endocarditis. J Am Coll Cardiol. 2017;69(3):325-44. 